\title{
Approximate Dynamic Programming Using Support Vector Regression
}

\author{
Brett Bethke, Jonathan P. How, and Asuman Ozdaglar
}

\begin{abstract}
This paper presents a new approximate policy iteration algorithm based on support vector regression (SVR). It provides an overview of commonly used cost approximation architectures in approximate dynamic programming problems, explains some difficulties encountered by these architectures, and argues that SVR-based architectures can avoid some of these difficulties. A key contribution of this paper is to present an extension of the SVR problem to carry out approximate policy iteration by minimizing the Bellman error at selected states. The algorithm does not require trajectory simulations to be performed and is able to utilize a rich set of basis functions in a computationally efficient way. A proof of the algorithm's correctness in the limit of sampling the entire state space is presented. Finally, computational results for two test problems are shown.
\end{abstract}

\section{INTRODUCTION}

Dynamic programming is a framework for addressing problems involving sequential decision making under uncertainty [1]. Such problems occur frequently in a number of fields, including engineering, finance, and operations research. This paper considers the general class of infinite horizon, discounted, finite state Markov Decision Processes (MDPs). The MDP is specified by $(\mathscr{S}, \mathscr{A}, P, g)$, where $\mathscr{S}$ is the state space, $\mathscr{A}$ is the action space, $P_{i j}(u)$ gives the transition probability from state $i$ to state $j$ under action $u$, and $g(i, u)$ gives the cost of taking action $u$ in state $i$. Future costs are discounted by a factor $0<\alpha<1$. A policy of the MDP is denoted by $\mu: \mathscr{S} \rightarrow \mathscr{A}$. Given the MDP specification, the problem is to minimize the so-called costto-go function $J_{\mu}$ over the set of admissible policies $\Pi$ :

$$
\min _{\mu \in \Pi} J_{\mu}\left(x_{0}\right)=\min _{\mu \in \Pi} \mathbb{E}\left[\sum_{k=0}^{\infty} \alpha^{k} g\left(x_{k}, \mu\left(x_{k}\right)\right)\right] .
$$

While MDPs are a powerful and general framework, they suffer from the well-known "curse of dimensionality", which states that as the problem size increases, the amount of computation necessary to find the solution increases exponentially rapidly. Indeed, for most MDPs of interest in the real world, this difficulty renders them impossible to solve exactly. To overcome the curse of dimensionality, researchers have investigated a number of methods for generating approximate solutions to large dynamic programs, giving rise to fields such as approximate dynamic programming, reinforcement learning, and neuro-dynamic programming. An

B. Bethke is a PhD Candidate, Dept. of Aeronautics and Astronautics, MIT, Cambridge, MA 02139, USA, bbethke@mit.edu

J. How is a Professor in the Dept. of Aeronautics and Astronautics, Massachusetts Institute of Technology, jhow@mit.edu

A. Ozdaglar is an Associate Professor in the Dept. of Electrical Engineering and Computer Science, Massachusetts Institute of Technology, asumanemit.edu important technique employed in many of these methods is the use of a parametric function approximation architecture to approximate the cost-to-go function $J_{\mu}$ of a given policy $\mu$. Once the cost-to-go function (or a suitable approximation thereof) is known, an improved policy can usually be computed. This process of policy evaluation followed by policy improvement is then repeated, yielding a method known as approximate policy iteration which produces a sequence of potentially improving policies [2].

A important problem in these approximate policy iteration methods is the choice of the cost function approximation architecture employed. The approximation is denoted by $\widetilde{J}_{\mu}(i ; \underline{\theta})$, where $i \in \mathscr{S}$ is a chosen state and $\underline{\theta}$ is a vector of tunable parameters. Numerous approximation architectures have already been investigated. Neural networks have received much attention as a useful approximation architecture. For example, Tesauro used a neural network approach in his famous TD-Gammon computer backgammon player, which was able to achieve world-class play [3], [4]. The linear combination of basis functions approach [1], [2], [5]-[9] has also been investigated extensively. In this approach, the designer picks a set of $r$ basis functions $\phi_{k}(i), k \in\{1 \ldots r\}$. The approximation $\widetilde{J}_{\mu}(i ; \underline{\theta})$ is then given by a linear combination of the basis functions,

$$
\widetilde{J}_{\mu}(i ; \underline{\theta})=\sum_{k=1}^{r} \theta_{k} \phi_{k}(i)=\underline{\theta}^{T} \underline{\phi}(i) .
$$

Once an approximation architecture is selected, the values of the tunable parameters $\underline{\theta}$ can be chosen in many ways [1, Vol II, Chapter 6]. So-called direct or simulation-based methods use simulation of state trajectories and the resulting trajectory costs to update the parameters. Another approach, known as Bellman error methods [2, Chapter 6, Section 10], attempt to choose the parameters by minimizing the Bellman error over a set of sample states $\mathscr{S}_{s}$ :

$\min _{\underline{\theta}} \sum_{i \in \mathscr{S}_{s}}\left(\widetilde{J}_{\mu}(i ; \underline{\theta})-\left(g(i, \mu(i))+\alpha \sum_{j \in \mathscr{S}} P_{i j}(\mu(i)) \widetilde{J}_{\mu}(j ; \underline{\theta})\right)\right)^{2}$

\section{A. Motivation for Support Vector Techniques}

Unfortunately, there are several difficulties associated with both the choice of approximation architecture and the method used to select the parameters. In both the neural network and basis function approximation architectures, the designer must trade off the expressiveness of the architecture (which may be thought of as the set of functions it can reproduce perfectly) with the need to maintain computational tractability. The former consideration pushes the design toward architectures with a large number of parameters. Indeed, a lookup table 
approximation architecture with $n=|\mathscr{S}|$ parameters could represent any arbitrary function $J_{\mu}($.$) perfectly by simply$ storing the value of the function at each of the $n$ states. Computational considerations require the use of fewer parameters, since implementing any architecture with a large number of parameters renders the resulting computations difficult to perform.

In addition to the issue regarding the number of parameters, each of the approximation architectures discussed so far presents its own unique difficulties. In the case of neural networks, the optimization problem that must be solved in the training process is nonconvex, which may lead to implementation difficulties. In addition, there can be ambiguity in the proper choice of the topology of the network (e.g., how many hidden layers to choose, how many nodes). Typically, experimentation is necessary to properly "tune" a neural network to a particular problem [10], [11]. In a basis function architecture, the set of basis functions themselves must be chosen, which may be difficult to do unless the designer has some prior knowledge about the structure of the true cost-to-go $J_{\mu}($.$) [8], [12]. If a poor set of basis functions$ is chosen, the architecture may be unable to approximate $J_{\mu}($.$) well even if the number of functions is large.$

Furthermore, simulation-based methods for updating the parameters of the chosen approximation architecture suffer from the simulation noise problem [2, Chapter 6]. This problem arises because of the need to sample state trajectories from the Markov chain associated with a given policy $\mu$. Simulation noise refers to the fact that there is randomness in the states and associated costs that will be observed in a given trajectory, which may ultimately lead to inaccurate estimates of the cost-to-go function. In practice, it may be necessary to simulate a very large number of trajectories, leading to increased computation time, in order to gain confidence that the resulting cost estimates are accurate. In contrast, Bellman error methods avoid the use of simulations by working directly with the Bellman equation. However, like simulation-based methods, they still suffer from the difficulties associated with the choice of approximation architecture discussed above.

To address these issues, we propose a different approximation architecture based on the idea of support vector regression (SVR) [10], [13]. SVR is similar in form to the basis function approach in that the approximation is given by a linear combination of basis functions (also called features in this context) as in Eq. (1). However, we will demonstrate that SVR has a number of advantages over both neural network and basis function architectures in the approximate dynamic programming problem, including:

- Being a kernel-based method, SVR can handle very large, possibly infinite, numbers of basis functions in a computationally tractable way. This makes the SVR architecture very expressive, yet practical to apply from a computational standpoint.

- The SVR solution is calculated via a convex quadratic program which has a unique optimal solution (contrast this with the nonconvex problem that arises in training a neural network).

- The difficulties of choosing network topologies, activation functions, and basis function sets are eliminated. Instead, the designer needs to select an appropriately powerful kernel (which implicitly specifies the basic function set). Many powerful yet easily computable kernels are known [14, Chapter 4].

The simplest use of the SVR architecture employs a simulation-based method to choose the parameters of the architecture. In this paper, we show that it is also possible to develop an SVR-based method that is similar in spirit to traditional Bellman error methods. This SVR-based method retains the advantage possessed by traditional Bellman error methods of not requiring trajectory simulations, while eliminating the difficulties associated with the choice of approximation architecture.

This paper presents this SVR-based method for approximate policy evaluation and proves its correctness in the limit of sampling the entire state space. The method is shown to be computationally efficient, and a full approximate policy iteration algorithm based on it is given. Finally, experimental results are provided that show that the algorithm converges quickly to a near-optimal policy in two test problems.

\section{Support VECTOR REgRESSION BASICS}

This section provides a basic overview of support vector regression; for more details, see [10]. The objective of the $\mathrm{SVR}$ problem is to learn a function

$$
f(x)=\sum_{k=1}^{r} \theta_{k} \phi_{k}(x)=\underline{\theta}^{T} \underline{\phi}(x)
$$

that gives a good approximation of a given set of training data $\left\{\left(x_{1}, y_{1}\right), \ldots,\left(x_{n}, y_{n}\right)\right\}$ where $x_{i} \in \mathbb{R}^{m}$ is the input data and $y_{i} \in \mathbb{R}$ is the observed output. Note that the functional form assumed for $f(x)$ is identical to Eq. (1). The training problem is posed as the following quadratic optimization problem:

$$
\begin{aligned}
\min _{\underline{\theta}, \underline{\xi}} \frac{1}{2}\|\underline{\theta}\|^{2} & +c \sum_{i=1}^{n}\left(\xi_{i}+\xi_{i}^{\star}\right) \\
\text { s.t. } \quad y_{i}-\underline{\theta}^{T} \underline{\phi}\left(x_{i}\right) & \leq \varepsilon+\xi_{i} \\
-y_{i}+\underline{\theta}^{T} \underline{\phi}\left(x_{i}\right) & \leq \varepsilon+\xi_{i}^{\star} \\
\xi_{i}, \xi_{i}^{\star} & \geq 0 \quad \forall i \in\{1, \ldots, n\} .
\end{aligned}
$$

Here, the regularization term $\frac{1}{2}\|\underline{\theta}\|^{2}$ penalizes model complexity, and the $\xi_{i}, \xi_{i}^{\star}$ are slack variables which are active whenever a training point $y_{i}$ lies farther than a distance $\varepsilon$ from the approximating function $f\left(x_{i}\right)$. The parameter $c$ trades off model complexity with accuracy of fitting the observed training data. As $c$ increases, any data points for which the slack variables are active incur higher cost, so the optimization problem tends to fit the data more closely (note that fitting too closely may not be desired if the training data is noisy).

The minimization problem [Eq. (2)] is difficult to solve when the number of basis functions $r$ is large, for two reasons. First, it is computationally demanding to compute the values of all $r$ basis functions for each of the data points. 
Second, the number of decision variables in the problem is $r$ (since there is one $\theta_{i}$ for each basis function $\phi_{i}($.$) ), so the$ minimization must be carried out in an $r$-dimensional space. To address these issues, one can solve the primal problem through its dual, which can be formulated by computing the Lagrangian and minimizing with respect to the primal variables $\underline{\theta}$ and $\xi_{i}, \xi_{i}^{\star}$ (again, for more details, see [10]). The dual problem is

$$
\begin{array}{r}
\max _{\underline{\lambda}, \underline{\lambda}^{\star}} \quad-\frac{1}{2} \sum_{i, i^{\prime}=1}^{n}\left(\lambda_{i}^{\star}-\lambda_{i}\right)\left(\lambda_{i^{\prime}}^{\star}-\lambda_{i^{\prime}}\right) \underline{\phi}\left(x_{i}\right)^{T} \underline{\phi}\left(x_{i^{\prime}}\right) \\
-\varepsilon \sum_{i=1}^{n}\left(\lambda_{i}^{\star}+\lambda_{i}\right)+\sum_{i=1}^{n} y_{i}\left(\lambda_{i}^{\star}-\lambda_{i}\right) \\
\text { s.t. } \quad 0 \leq \lambda_{i}, \lambda_{i}^{\star} \leq c \quad \forall i \in\{1, \ldots, n\} .
\end{array}
$$

Note that the basis function vectors $\phi\left(x_{i}\right)$ now appear only as inner products. This is important, because in many cases a kernel function $K\left(x_{i}, x_{i^{\prime}}\right)=\phi\left(x_{i}\right)^{T} \phi\left(x_{i^{\prime}}\right)$ can be defined whose evaluation avoids the need to explicitly calculate the vectors $\phi\left(x_{i}\right)$, resulting in significant computational savings. Also, the dimensionality of the dual problem is reduced to only $2 n$ decision variables, since there is one $\lambda_{i}$ and one $\lambda_{i}^{\star}$ for each of the data points. Again, when the number of basis functions is large, this results in significant computational savings. Furthermore, it is well known that the dual problem can be solved efficiently using techniques such as Sequential Minimal Optimization (SMO) [15], [16]. Many libraries such as libSVM [17] implement SMO to solve the SVR problem (i.e. find the values of the dual variables $\left.\underline{\lambda}, \underline{\lambda}^{\star}\right)$. Once the dual variables are known, the function $f(x)$ can be computed using the so-called support vector expansion:

$$
f(x)=\sum_{i=1}^{n}\left(\lambda_{i}-\lambda_{i}^{\star}\right) \underline{\phi}\left(x_{i}\right)^{T} \underline{\phi}(x)=\sum_{i=1}^{n}\left(\lambda_{i}-\lambda_{i}^{\star}\right) K\left(x_{i}, x\right) .
$$

\section{SVR-BASEd POLICy EVAluation}

Given the advantages outlined above of an SVR-based cost approximation architecture, this section now presents a progression of ideas on how to incorporate SVR into the approximate policy evaluation problem.

\section{A. Formulation 1: Simulation-based Approach}

The first formulation is similar in spirit to simulation-based methods such as $\operatorname{TD}(\lambda)[18]$ and $\operatorname{LSPE}(\lambda)$ [7]. In particular, a number of sample states $\mathscr{S}_{s}$ are chosen, and many trajectories are simulated starting at these states. The costs for each state $i$ are averaged over the trajectories to give an approximation $\hat{J}_{i}$ to the cost-to-go, the training data $\left\{\left(i, \hat{J}_{i}\right) \mid i \in \mathscr{S}_{s}\right\}$ are taken as the input to the SVR training problem, and an approximating function $\widetilde{J}_{\mu}(i ; \underline{\theta})$ is calculated by solving the basic SVR problem.

\section{B. Formulation 2: Simulation-Free Approach Using Bellman Error}

The approach of Formulation 1 leverages the power of the SVR architecture and allows the use of high-dimensional feature vectors in a computationally efficient way, which is an attractive advantage. However, it still requires simulation of multiple trajectories per state, which may be time-consuming and also introduces undesirable simulation noise into the problem.

Fortunately, the SVR-based approach can be reformulated to eliminate the need for trajectory simulation. To do this, first recall the standard Bellman equation for evaluating a given policy $\mu$ exactly. Writing the exact solution for the cost-to-go as a vector $\underline{J}_{\mu} \in \mathbb{R}^{n}$, where $n=|\mathscr{S}|$ is the size of the state space, the Bellman equation is

$$
\underline{J}_{\mu}=T_{\mu} \underline{J}_{\mu}=\underline{g}+\alpha P^{\mu} \underline{J}_{\mu}
$$

where

$$
P_{i j}^{\mu} \equiv P_{i j}(\mu(i))
$$

is the probability transition matrix associated with $\mu$, and

$$
g_{i} \equiv \sum_{j \in \mathscr{S}} P_{i j}^{\mu} g(i, \mu(i), j)
$$

is the expected single stage cost of the policy $\mu$ starting from state $i$. The Bellman equation given by Eq. (6) is a linear system in $\underline{J}_{\mu}$, and can be solved exactly:

$$
\underline{J}_{\mu}=\left(I-\alpha P^{\mu}\right)^{-1} \underline{g} .
$$

In practice, of course, the size of the state space is much too large to admit solving the Bellman equation exactly, which is why approximation methods must be used. Note, however, that the Bellman equation still provides useful information even in the approximate setting. In particular, a candidate approximation function $\widetilde{J}_{\mu}($.$) should be "close" to satisfying$ the Bellman equation if it is truly a good approximation. To quantify this notion, define the Bellman error $B E(i)$ as

$$
B E(i) \equiv \widetilde{J}_{\mu}(i)-\left(g_{i}+\alpha \sum_{j \in \mathscr{S}} P_{i j}^{\mu} \widetilde{J}_{\mu}(j)\right),
$$

which is a measure of how well the approximation function $\widetilde{J_{\mu}}(i)$ solves Eq. (6), and therefore, how well the approximation reflects the true cost. Note that $B E(i)$ is a function of the state $i$, and ideally, $|B E(i)|$ should be as small as possible for as many states as possible. In particular, note that if $B E(i)=0$ for every state, then by definition $\widetilde{J}_{\mu}(i)=J_{\mu}(i)$ at every state; that is, $\widetilde{J}_{\mu}(i)$ is exact.

Recall that the SVR problem seeks to minimize the absolute value of some error function (mathematically, this is stated as the constraints Eqs. (3) and (4) in the optimization problem). In Formulation 1, the error function is $\left(\hat{J_{i}}-\right.$ $\left.\widetilde{J}_{\mu}(i ; \underline{\theta})\right)$; that is, the difference between the simulated cost values and the approximation. Note that this formulation is an indirect approach which generates an approximation function $\widetilde{J_{\mu}}($.$) , which in turn hopefully keeps the Bellman error small$ at many states. The key idea behind Formulation 2 is that the SVR optimization problem can be modified to minimize the Bellman error directly, without the need to simulate trajectories. The change to the optimization problem is simple: the Bellman error is substituted [Eq. (9)] as the error 
function in the normal SVR problem:

$$
\begin{aligned}
\min _{\underline{\theta}, \underline{\xi}} \frac{1}{2}\|\underline{\theta}\|^{2} & +c \sum_{i \in \mathscr{S}_{s}}\left(\xi_{i}+\xi_{i}^{\star}\right) \\
\text { s.t. } \quad B E(i) & \leq \varepsilon+\xi_{i} \\
-B E(i) & \leq \varepsilon+\xi_{i}^{\star} \\
\xi_{i}, \xi_{i}^{\star} & \geq 0 \quad \forall i \in \mathscr{S}_{s} .
\end{aligned}
$$

This optimization problem is referred to as the Bellman error optimization problem. Note that by substituting the functional form of $\widetilde{J}_{\mu}$ [Eq. (1)] into Eq. (9), the Bellman error can be written

$$
\begin{aligned}
B E(i) & =\underline{\theta}^{T} \underline{\phi}(i)-\left(g_{i}+\alpha \sum_{j \in \mathscr{S}} P_{i j}^{\mu}\left(\underline{\theta}^{T} \underline{\phi}(j)\right)\right) \\
& =-g_{i}+\underline{\theta}^{T} \underline{\psi}(i),
\end{aligned}
$$

where

$$
\underline{\psi}(i) \equiv \underline{\phi}(i)-\alpha \sum_{j \in \mathscr{S}} P_{i j}^{\mu} \underline{\phi}(j) .
$$

Therefore, the Bellman error optimization problem can be rewritten as

$$
\begin{aligned}
\min _{\underline{\theta}, \underline{\xi}} \frac{1}{2}\|\underline{\theta}\|^{2} & +c \sum_{i \in \mathscr{S}_{s}}\left(\xi_{i}+\xi_{i}^{\star}\right) \\
\text { s.t. } \quad-g_{i}+\underline{\theta}^{T} \underline{\psi}(i) & \leq \varepsilon+\xi_{i} \\
g_{i}-\underline{\theta}^{T} \underline{\psi}(i) & \leq \varepsilon+\xi_{i}^{\star} \\
\xi_{i}, \xi_{i}^{\star} & \geq 0 \quad \forall i \in \mathscr{S}_{s} .
\end{aligned}
$$

This problem is identical in form to the basic support vector regression problem [Eq. (2)]. The only difference is that the feature vector $\phi(i)$ has been replaced by a new feature vector $\psi(i)$; this amounts to simply using a different set of basis functions and does not alter the structure of the basic problem in any way. Therefore, the dual problem is given by Eq. (5) with the substitution $\underline{\phi}(i) \rightarrow \underline{\psi}(i)$ :

$$
\begin{array}{r}
\max _{\underline{\lambda}, \underline{\lambda}^{\star}}-\frac{1}{2} \sum_{i, i^{\prime}=1}^{n}\left(\lambda_{i}^{\star}-\lambda_{i}\right)\left(\lambda_{i^{\prime}}^{\star}-\lambda_{i^{\prime}}\right) \underline{\psi}(i)^{T} \underline{\psi}\left(i^{\prime}\right) \\
-\varepsilon \sum_{i=1}^{n}\left(\lambda_{i}^{\star}+\lambda_{i}\right)+\sum_{i=1}^{n} g_{i}\left(\lambda_{i}^{\star}-\lambda_{i}\right) \\
\text { s.t. } \quad 0 \leq \lambda_{i}, \lambda_{i}^{\star} \leq c \quad \forall i \in \mathscr{S}_{s} .
\end{array}
$$

In order to solve the dual problem, the $g_{i}$ values and the kernel values $\mathscr{K}\left(i, i^{\prime}\right)=\psi(i)^{T} \psi\left(i^{\prime}\right)$ must be computed. The $g_{i}$ values are given by Eq. (7), and a simple calculation using Eq. (10) yields a formula for $\mathscr{K}\left(i, i^{\prime}\right)$ :

$$
\begin{gathered}
\mathscr{K}\left(i, i^{\prime}\right)=K\left(i, i^{\prime}\right)-\alpha \sum_{j \in \mathscr{S}}\left(P_{i^{\prime} j}^{\mu} K(i, j)+P_{i j}^{\mu} K\left(i^{\prime}, j\right)\right) \\
+\alpha^{2} \sum_{j, j^{\prime} \in \mathscr{S}} P_{i j}^{\mu} P_{i^{\prime} j^{\prime}}^{\mu} K\left(j, j^{\prime}\right) .
\end{gathered}
$$

Here, $K\left(i, i^{\prime}\right)=\phi(i)^{T} \phi\left(i^{\prime}\right)$ is the kernel function corresponding to the feature vectors $\phi(i)$. Note that, similar to the basic SVR problem, significant computational savings can be achieved by using a closed-form kernel function which avoids the need to explicitly evaluate the feature vectors $\underline{\phi}(i)$.
Once the kernel values $\mathscr{K}\left(i, i^{\prime}\right)$ and the cost values $g_{i}$ are computed, the dual problem is completely specified and can be solved (e.g., using a standard SVM solving package such as libSVM [17]), yielding the dual solution variables $\underline{\lambda}$. Again, in direct analogy with the normal SV regression problem, the primal variables $\underline{\theta}$ are given by the relation

$$
\underline{\theta}=\sum_{i \in \mathscr{S}_{s}}\left(\lambda_{i}-\lambda_{i}^{\star}\right) \underline{\psi}(i) .
$$

Substituting this expression for $\underline{\theta}$ into Eq. (1) yields

$$
\begin{aligned}
\widetilde{J}_{\mu}(k) & =\underline{\theta}^{T} \underline{\phi}(k) \\
& =\sum_{i \in \mathscr{S}_{s}}\left(\lambda_{i}-\lambda_{i}^{\star}\right) \underline{\psi}(i)^{T} \underline{\phi}(k) \\
& =\sum_{i \in \mathscr{S}_{s}}\left(\lambda_{i}-\lambda_{i}^{\star}\right)\left(K(i, k)-\alpha \sum_{j \in \mathscr{S}} P_{i j}^{\mu} K(j, k)\right)
\end{aligned}
$$

Thus, once the dual variables $\underline{\lambda}$ are known, Eq. (15) can be used to calculate $\widetilde{J}_{\mu}(k)$.

\section{Formulation 3: Forcing the Bellman Error to Zero}

Formulation 2 performs approximate policy evaluation without having to resort to trajectory simulation, thereby eliminating simulation noise. A final reformulation is possible by considering the settings of the SVR parameters $c$ and $\varepsilon$. Recall that, in the standard SVR problem, the choice of $c$ and $\varepsilon$ are related to how noisy the input data is. In a simulation-based method, simulation noise introduced into the problem must be considered. The situation in Formulation 2, however, is fundamentally different: there is no noise in the problem. That is, instead of observing noisy simulation data generated by sampling the underlying random Markov chain associated with the policy $\mu$, we are working directly with the Bellman equation, which is an exact description of a mathematical relation between the cost-to-go values $J_{\mu}(i)$ for all states $i \in \mathscr{S}$. Therefore, it is reasonable to explicitly require that the Bellman error be exactly zero at the sampled states, which can be accomplished by setting $\varepsilon=0$ and $c=\infty$.

Having fixed $c$ and $\varepsilon$, the optimization problem of Formulation 2 [Eq. (11)] can be recast in a simpler form. With $c=\infty$, the optimal solution must have $\xi, \xi^{\star}=0$ for all $i$, since otherwise the objective function will be unbounded. Therefore, any feasible solution must have $\xi, \xi^{\star}=0$. Furthermore, if $\varepsilon$ is also zero, then the constraints [Eqs. (12) and (13)] become equalities. With these modifications, the primal problem reduces to

$$
\begin{array}{ll} 
& \min _{\underline{\theta}} \frac{1}{2}\|\underline{\theta}\|^{2} \\
\text { s.t. } & g_{i}-\underline{\theta}^{T} \underline{\psi}(i)=0 \quad \forall i \in \mathscr{S}_{s},
\end{array}
$$

where $\psi(i)$ is given by Eq. (10). The Lagrange dual of this optimization problem is easily calculated. The Lagrangian is

$$
\mathscr{L}(\underline{\theta}, \underline{\lambda})=\frac{1}{2}\|\underline{\theta}\|^{2}+\sum_{i \in \mathscr{S}_{s}} \lambda_{i}\left(g_{i}-\underline{\theta}^{T} \underline{\psi}(i)\right) .
$$


Maximizing $\mathscr{L}(\underline{\theta}, \underline{\lambda})$ with respect to $\underline{\theta}$ can be accomplished by setting the corresponding partial derivative to zero:

$$
\frac{\partial \mathscr{L}}{\partial \underline{\theta}}=\underline{\theta}-\sum_{i} \lambda_{i} \underline{\psi}(i)=0,
$$

and therefore

$$
\underline{\theta}=\sum_{i} \lambda_{i} \underline{\psi}(i)
$$

Thus, the approximation $\widetilde{J}_{\mu}(i)$ is given by

$$
\begin{aligned}
\widetilde{J}_{\mu}(k) & =\underline{\theta}^{T} \underline{\phi}(k) \\
& =\sum_{i \in \mathscr{S}_{s}} \lambda_{i} \underline{\psi}(i)^{T} \underline{\phi}(k) \\
& =\sum_{i \in \mathscr{S}_{s}} \lambda_{i}\left(K(i, k)-\alpha \sum_{j \in \mathscr{S}} P_{i j}^{\mu} K(j, k)\right) .
\end{aligned}
$$

Finally, substituting Eq. (18) into Eq. (17) and maximizing with respect to all $\lambda_{i}$ variables gives the dual problem:

$$
\max _{\underline{\lambda} \in \mathbb{R}^{n}}-\frac{1}{2} \sum_{i, i^{\prime}} \lambda_{i} \lambda_{i^{\prime}} \underline{\psi}(i)^{T} \underline{\psi}\left(i^{\prime}\right)+\sum_{i} \lambda_{i} g_{i} .
$$

This can also be written in vector form as

$$
\max _{\underline{\lambda} \in \mathbb{R}^{n}}-\frac{1}{2} \underline{\lambda^{T}} \mathbb{K} \underline{\lambda}+\underline{\lambda}^{T} \underline{g},
$$

where $\mathbb{K}_{i i^{\prime}}=\psi(i)^{T} \psi\left(i^{\prime}\right)=\mathscr{K}\left(i, i^{\prime}\right)$ is the Gram matrix of the kernel $\mathscr{K}(.,$.$) (which is calculated using Eq. (14)). Note$ that the problem is a simple, unconstrained maximization of a quadratic form which has a unique maximum since $\mathbb{K}$ is positive definite. The solution is found analytically by setting the derivative of the objective with respect to $\underline{\lambda}$ to zero, which yields

$$
\mathbb{K} \underline{\lambda}=\underline{g} .
$$

Note the important fact that the dimension of this linear system is $n_{s}=\left|\mathscr{S}_{s}\right|$, the number of sampled states. Recall that the original problem of calculating the exact solution $\underline{J}_{\mu}$ [Eq. (8)] involved solving a linear system in $n$ variables, where $n$ is the size of the entire state space (which, by assumption, is very large and makes the solution of the original system difficult to find). After developing a support vector regression-based method for approximating the true cost-to-go and reformulating it based on parameter selection observations, the approximation problem has been reduced to a problem of solving another linear system [Eq. (21)]. However, this time the system is in $n_{s}=\left|\mathscr{S}_{s}\right|$ variables, where $n_{s} \ll n$. Furthermore, since the designer is in control of $\mathscr{S}_{s}$, they can select the number of sample states based on the computational resources available.

\section{Support Vector Policy Iteration Algorithm}

The preceding section showed how to construct a cost-togo approximation $\widetilde{J}_{\mu}($.$) of a fixed policy \mu$. This section now presents a full support vector policy iteration algorithm.

Step 1 (Preliminary) Choose a kernel function $K\left(i, i^{\prime}\right)$ defined on $\mathscr{S} \times \mathscr{S}$.
Step 2 (Preliminary) Select a subset of states $\mathscr{S}_{s} \subset \mathscr{S}$ to sample. The cardinality of $\mathscr{S}_{s}$ should be based on the computational capability available but will certainly be much smaller than $|\mathscr{S}|$ for a large problem.

Step 3 (Preliminary) Select an initial policy $\mu_{0}$.

Step 4 (Policy evaluation) Given the current policy $\mu_{k}$, calculate the kernel Gram matrix $\mathbb{K}$ for all $i, i^{\prime} \in \mathscr{S}_{s}$ using Eq. (14). Also calculate the cost values $g_{i}$ using Eq. (7).

Step 5 (Policy evaluation) Using the values calculated in Step 4, solve the linear system [Eq. (21)] for $\underline{\lambda}$.

Step 6 (Policy evaluation) Using the dual solution variables $\underline{\lambda}$, construct the cost-to-go approximation $\widetilde{J}_{\mu_{k}}(i)$ using Eq. (19).

Step 7 (Policy improvement) Using the cost-to-go $\widetilde{J}_{\mu_{k}}(i)$ found in Step 6, calculate the one-step policy improvement $T_{\mu_{k+1}} \widetilde{J}_{\mu_{k}}=T \widetilde{J}_{\mu_{k}}$ :

$$
\mu_{k+1}(i)=\arg \min _{u} \sum_{j \in \mathscr{S}} P_{i j}(u)\left(g(i, u)+\alpha \widetilde{J}_{\mu_{k}}(j)\right)
$$

Step 8 Set the current policy $\mu=\mu_{k+1}$ and go back to Step 4.

\section{A. Proof of Exactness when $\mathscr{S}_{s}=\mathscr{S}$}

Under certain assumptions about the chosen feature mapping $\phi(i)$ and the associated kernel function $K\left(i, i^{\prime}\right)=$ $\phi(i)^{T} \bar{\phi}\left(i^{\prime}\right)$, the support vector policy iteration algorithm gives the same results as exact policy iteration in the limit of sampling the entire state space. A proof of this result is provided in this section. The proof relies on the following lemma.

Lemma: Assume the feature vectors $\{\phi(i) \mid i \in \mathscr{S}\}$ are linearly independent. Then the vectors $\left\{\underline{\psi}(i) \mid i \in \mathscr{S}_{s}\right\}$, where $\underline{\psi}(i)=\underline{\phi}(i)-\alpha \sum_{j \in \mathscr{S}} P_{i j}^{\mu} \underline{\phi}(j)$, are also linearly independent.

Proof: Consider the vector space $\mathscr{V}$ spanned by the vectors $\{\phi(i) \mid i \in \mathscr{S}\}$. It is clear that $\psi(i)$ is a linear combination of vectors in $\mathscr{V}$, so a linear operator $A$ that maps $\phi(i)$ to $\underline{\psi}(i)$ can be defined:

$$
\underline{\psi}(i)=A \underline{\phi}(i)=\left(I-\alpha P^{\mu}\right) \underline{\phi}(i)
$$

Here, $I$ is the identity matrix and $P^{\mu}$ is the probability transition matrix for the policy $\mu$. Since $P^{\mu}$ is a stochastic matrix, its largest eigenvalue is 1 and all other eigenvalues have absolute value less than 1 ; hence all eigenvalues of $\alpha P^{\mu}$ have absolute value less than or equal to $\alpha<1$. Since all eigenvalues of $I$ are equal to 1 , the linear operator $A=I-\alpha P^{\mu}$ is full rank and $\operatorname{dim}(\operatorname{ker}(A))=0$. Therefore,

$$
\operatorname{dim}\left(\left\{\underline{\psi}(i) \mid i \in \mathscr{S}_{s}\right\}\right)=\operatorname{dim}\left(\left\{\underline{\phi}(i) \mid i \in \mathscr{S}_{s}\right\}\right)=n_{s}
$$

so the vectors $\left\{\psi(i) \mid i \in \mathscr{S}_{s}\right\}$ are linearly independent.

The following now presents the main theorem:

Theorem 1: Assume the feature vectors $\{\underline{\phi}(i) \mid i \in \mathscr{S}\}$ are linearly independent, and let $\mathscr{S}_{s}=\mathscr{S}$. Then the approximation $\widetilde{J}_{\mu}($.$) calculated by the support vector policy iteration$ algorithm is exact; that is, $\widetilde{J}_{\mu}(i)=J_{\mu}(i)$ for all $i \in \mathscr{S}$.

Proof: Since the feature vectors $\left\{\phi(i) \mid i \in \mathscr{I}_{s}\right\}$ are linearly independent, so are $\left\{\psi(i) \mid i \in \mathscr{S}_{s}\right\}$ (by the Lemma). 
Therefore, the Gram matrix $\mathbb{K}$, where $\mathbb{K}_{i i^{\prime}}=\mathscr{K}\left(i, i^{\prime}\right)=$ $\underline{\psi}(i)^{T} \underline{\psi}\left(i^{\prime}\right)$, is full rank and invertible, so Eq. (21) has a unique solution for $\underline{\lambda}$. Having found a feasible solution $\underline{\lambda}$ for the dual problem [Eq. (20)], Slater's condition is satisfied and strong duality holds. Therefore, the primal problem [Eq. (16)] is feasible, and its optimal solution is given by Eq. (18). Feasibility of this solution implies

$$
\begin{aligned}
B E(i) & =-g_{i}+\underline{\theta}^{T} \underline{\psi}(i) \\
& =-g_{i}+\underline{\theta}^{T}\left(\underline{\phi}(i)-\alpha \sum_{j \in \mathscr{S}} P_{i j}^{\mu} \underline{\phi}(j)\right) \\
& =\widetilde{J}_{\mu}(i)-\left(g_{i}+\alpha \sum_{j \in \mathscr{S}} P_{i j}^{\mu} \widetilde{J}_{\mu}(j)\right) \\
& =0 \quad \forall i \in \mathscr{S}
\end{aligned}
$$

Therefore, the Bellman equation is satisfied at every state $i \in \mathscr{S}$ by the function $\widetilde{J}_{\mu}$, so

$$
\widetilde{J}_{\mu}(i)=J_{\mu}(i) \quad \forall i \in \mathscr{S}
$$

Theorem 1 shows that the support vector policy iteration algorithm reduces to exact policy iteration when the entire state space is sampled. This result is encouraging, since it is well known that exact policy iteration converges to the optimal policy in a finite number of steps [1]. Also, note that even when only part of the entire state space is sampled, the Bellman error at the sampled states is still exactly zero as long as a solution to Eq. (21) can be found.

\section{B. Kernel Selection}

The choice of kernel function $K\left(i, i^{\prime}\right)$ is an important design consideration, since it determines the general structure of the approximation architecture [Eq. (19)]. A common choice is the radial basis function (RBF) kernel

$$
K\left(i, i^{\prime}\right)=\exp \left(-\left\|i-i^{\prime}\right\|^{2} / \gamma\right)
$$

This kernel depends on the existence of a suitable distance metric $\left\|i-i^{\prime}\right\|$ on the state space, as well as the characteristic length-scale $\gamma$. It can be shown [14, Section 4.2.1] that the feature vectors corresponding to the RBF kernel are infinitedimensional. Another possibility is the polynomial kernel

$$
K\left(i, i^{\prime}\right)=\left(1+<i, i^{\prime}>\right)^{p}
$$

which corresponds to the $p$-order polynomial feature vectors $\phi(i)=\left(1, i, \ldots, i^{p}\right)^{T}$. There are many other possible choices for the kernel. Furthermore, new kernels can be constructed by combining the feature vectors from a set of primitive kernels (allowing one, for example, to augment a kernel with features known from previous experience). For an overview of the kernel selection problem, see [13, Chapter 13], [14, Chapter 4].

\section{Numerical Results}

The support vector policy iteration method was implemented on two test problems to evaluate its performance.

\section{A. Test 1}

The first test problem was a one dimensional, deterministic problem with state space $\mathscr{S}=$ $\{-150.0,-149.9, \ldots, 149.9,150.0\}$, so that $n=|\mathscr{S}|=3000$. In this problem, the action space is the same as the state space $(\mathscr{A}=\mathscr{S})$, and the system dynamics are given by

$$
x_{k+1}=x_{k}+u_{k}
$$

The cost function is a nonlinear and discontinuous function of the state $x$ :

$$
g(x, u)= \begin{cases}(x+75)^{2}+10 u^{2} & x<0 \\ (x-75)^{2}+10 u^{2} & 0 \leq x<5 \\ 5(x-75)^{2}+10 u^{2} & x \geq 5\end{cases}
$$

This cost function was chosen to pose an intentionally challenging problem to the support vector policy iteration algorithm, since the resulting optimal cost-to-go function $J^{\star}(x)$ also turns out to be nonlinear and discontinuous (see Figure 1). The discount factor used was $\alpha=0.99$.

To implement the support vector policy iteration algorithm, a radial basis function kernel $K\left(x, x^{\prime}\right)=\exp (-(x-$ $\left.\left.x^{\prime}\right)^{2} / 50\right)$ was used, and an equally spaced grid of seven sample points, $\mathscr{S}_{s}=\{-150,-100,-50,0,50,100,150\}$, was selected. The algorithm was then executed, yielding a sequence of policies and associated cost functions that converged after 5 iterations. Figure 1 shows the convergence of the cost functions generated by the algorithm, shown in green, relative to the true optimal cost $J^{\star}(x)$, shown in blue.

Notice that the number of chosen sample points (7), and therefore the amount of computation required for the approximate policy iteration algorithm, is nearly three orders of magnitude less than the total number of states (3000). Indeed, calculating the exact solution $J^{\star}(x)$ to the test problem using value iteration took over 15 minutes. In contrast, each iteration of the support vector policy iteration algorithm took less than a second, and the algorithm converged in approximately 4.5 seconds.

Of course, dramatic improvements in computational speed are useful only if the resulting approximate policy is close to the optimal policy found from the exact solution. Figure 2 compares the two policies. Despite "looking" at only 7 out of the 3000 states in the problem, the support vector policy iteration algorithm is able to generate a policy that is qualitatively and quantitatively similar to the optimal policy. Qualitatively, despite the strong discontinuity in both the policy and the optimal cost function introduced by the choice of the function $g(x, i)$, the approximate algorithm is able to capture the structure of both the optimal cost and the policy quite well. Quantitatively, the actual cost-to-go of the policy generated by the support vector policy iteration algorithm was computed exactly (this was feasible to do due to the relatively small size of the state space) and compared to the optimal cost-to-go at each state. Averaged over all states, the cost of the approximate policy was only $4.5 \%$ higher than the optimal cost. This difference between the optimal and approximate policies is called the policy loss. 
TABLE I
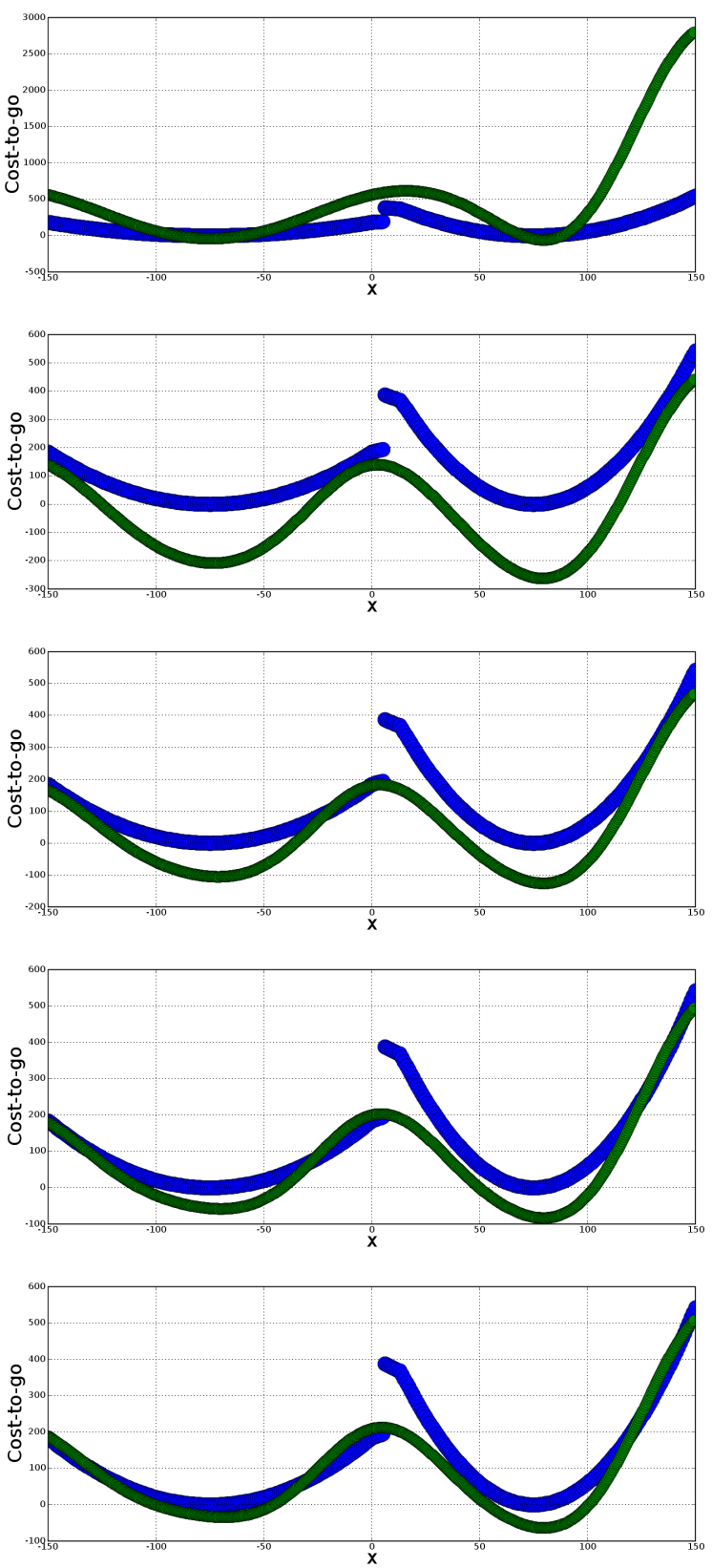

Fig. 1. Support vector policy iteration on a test problem. The approximate cost $\widetilde{J}_{\mu_{k}}(x)$ is shown in green, and the optimal cost $J^{\star}(x)$ is shown in blue. The support vector policy iteration algorithm converges in 5 iterations, shown top to bottom.

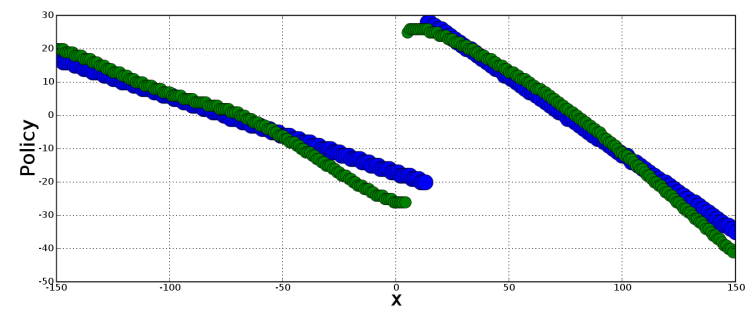

Fig. 2. Comparison of the optimal policy (blue) with the policy found by the support vector policy iteration algorithm (green) found in 5 iterations.
COMPUTATION TIME AND POLICY LOSS FOR NUMERICAL EXPERIMENTS

\begin{tabular}{c|c|c|c|c}
\hline & $\begin{array}{c}\text { Value iteration } \\
\text { runtime }\end{array}$ & $\begin{array}{c}\text { Support vector } \\
\text { policy iteration } \\
\text { runtime }\end{array}$ & $\begin{array}{c}\text { Computation } \\
\text { speedup }\end{array}$ & $\begin{array}{c}\text { Policy } \\
\text { loss }\end{array}$ \\
\hline \hline Test 1 & $900.0 \mathrm{~s}$ & $4.5 \mathrm{~s}$ & $\times 200$ & $4.5 \%$ \\
Test 2 & $29,592 \mathrm{~s}$ & $18.0 \mathrm{~s}$ & $\times 1643$ & $6.3 \%$ \\
\hline
\end{tabular}

\section{B. Test 2}

The second test problem was a two dimensional, deterministic problem with state vector $\underline{X}=(x, v)^{T}$ and state space

$$
\begin{aligned}
\mathscr{S}= & \{-80.0,-79.5, \ldots, 79.5,80.0\} \otimes \\
& \{-80.0,-79.5, \ldots, 79.5,80.0\},
\end{aligned}
$$

so that $n=|\mathscr{S}|=102,400$. The action space was $\mathscr{A}=$ $\{-2,-1.5, \ldots, 1.5,2\}$, and the system dynamics were

$$
\begin{aligned}
& x_{k+1}=x_{k}+v_{k} \\
& v_{k+1}=v_{k}+u_{k}
\end{aligned}
$$

The cost function was

$$
g(\underline{X}, u)=x^{2}+x^{4} / 80^{2}+10 u^{2}
$$

Again, the discount factor used was $\alpha=0.99$.

The kernel function used in this test was again a radial basis function kernel, $K\left(\underline{X}, \underline{X}^{\prime}\right)=\exp \left(-\left\|\underline{X}-\underline{X}^{\prime}\right\|^{2} / 80\right)$. A set of only 25 sample states was used; Figure 3 shows a graphical representation of the state space $\mathscr{S}$ as well as the sample states $\mathscr{S}_{s}$. Note that only $25 / 102,400=0.024 \%$ of the state space was sampled.

Due to the very large size of the state space, solving the problem exactly using value iteration took 493 minutes. In contrast, the support vector policy iteration algorithm converged after 7 iterations, requiring a total of only 18 seconds. Figure 4 shows a comparison of the optimal cost to the cost found by the support vector policy iteration algorithm for fixed $v=0$. Comparison of the optimal policy to the approximate policy over the entire state space reveals a policy loss of only $6.3 \%$.

The experimental results for both tests are summarized in Table I. With any approximate method, there is a tradeoff between computation speedup (i.e. the ratio of computation runtimes of the exact and approximate methods) versus the resultant policy loss. The table emphasizes the fact that the support vector policy iteration algorithm can provide significant speedup while keeping the policy loss small.

\section{CONCLUSION}

This paper has presented an approximate policy iteration algorithm based on support vector regression that avoids some of the difficulties encountered in other approximate dynamic programming methods. In particular, the approximation architecture used in this algorithm is kernel-based, allowing it to implicitly work with a very large set of basis functions. This is a significant advantage over other methods, 


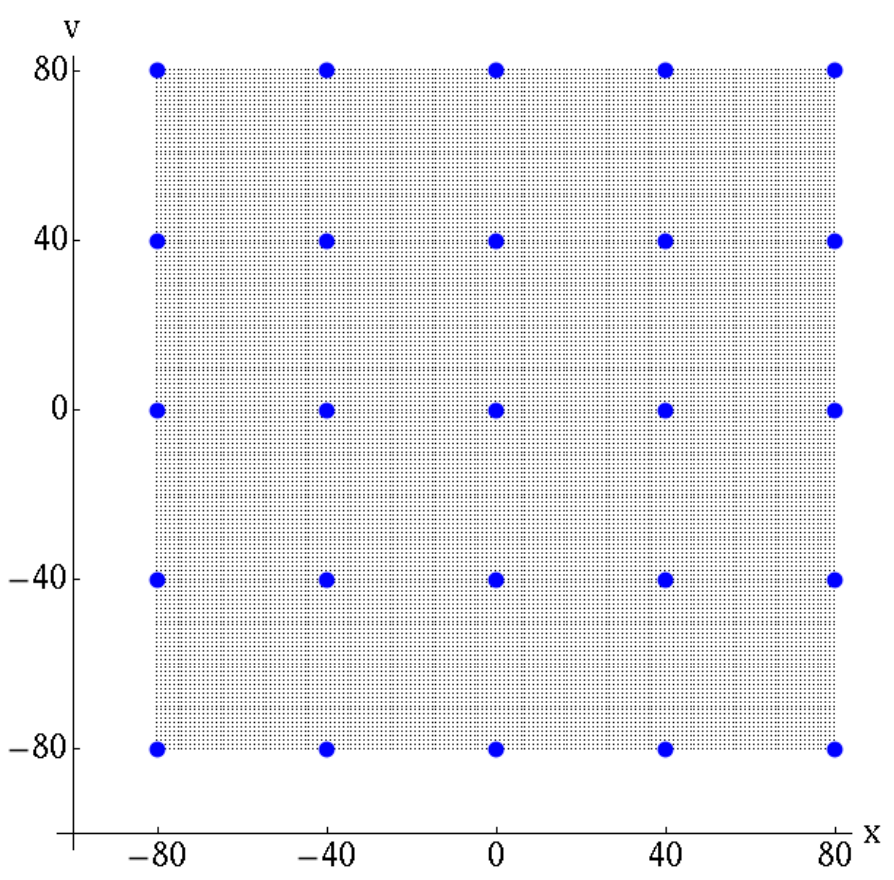

Fig. 3. Comparison of the full state space $\mathscr{S}$, shown as small black dots, versus the sample states $\mathscr{S}_{s}$, shown as large blue dots, in Test 2 . Only 25 of 102,400 , or $0.024 \%$, of the states were sampled.

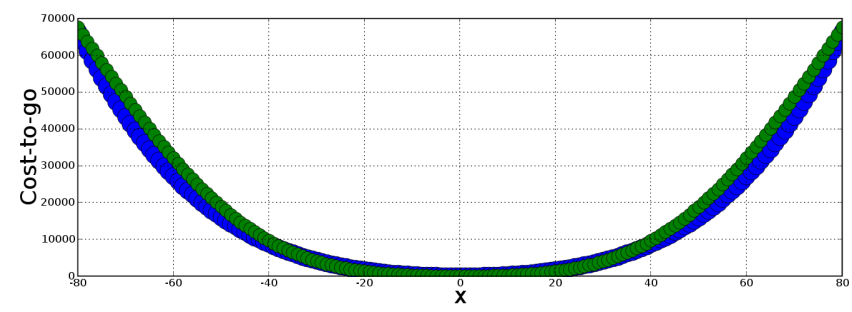

Fig. 4. Optimal cost-to-go $J^{\star}\left((x, 0)^{T}\right)$ (shown in blue) versus the final approximate cost-to-go $\widetilde{J}_{\mu_{k}}\left((x, 0)^{T}\right)$ generated by the support vector policy iteration algorithm (shown in green).

which encounter computational difficulties as the number of basis functions increases. In addition, the architecture is "trained" by solving a simple, convex optimization problem (unlike, for example, the neural network training process). Furthermore, the support vector policy iteration algorithm avoids simulations and the associated simulation noise problem by minimizing the Bellman error of the approximation directly. The algorithm has the attractive theoretical property of being provably exact in the limit of sampling the entire state space.

Experimental results of implementing the algorithm on two test problems indicate that it has a large computational advantage over computing the solution exactly using value iteration, while still yielding high-quality policy and cost approximations.

\section{ACKNOWLEDGEMENTS}

Research supported in part by the Boeing Company under the guidance of Dr. John Vian at the Boeing Phantom Works,
Seattle and by AFOSR grant FA9550-04-1-0458. The first author is also supported by the Hertz Foundation and the American Society for Engineering Education.

\section{REFERENCES}

[1] D. Bertsekas, Dynamic Programming and Optimal Control. Belmont, MA: Athena Scientific, 2007.

[2] D. Bertsekas, J. Tsitsiklis, Neuro-Dynamic Programming. Belmont, MA: Athena Scientific, 1996.

[3] G. Tesauro, "Temporal difference learning and TD-Gammon," Commun. ACM, vol. 38, no. 3, pp. 58-68, 1995.

[4] — - "Practical issues in temporal difference learning," Machine Learning, vol. 8, pp. 257-277, 1992.

[5] M. Lagoudakis and R. Parr, "Least-squares policy iteration," Journal of Machine Learning Research, vol. 4, pp. 1107-1149, 2003.

[6] W. P. J. Si, A. Barto and D. Wunsch, Learning and Approximate Dynamic Programming. NY: IEEE Press, 2004. [Online]. Available: http://citeseer.ist.psu.edu/651143.html

[7] D. Bertsekas and S. Ioffe, "Temporal Differences-Based Policy Iteration and Applications in Neuro-Dynamic Programming,' http://web.mit.edu/people/dimitrib/Tempdif.pdf, 1996.

[8] D.P. de Farias, B. Van Roy, "The Linear Programming Approach to Approximate Dynamic Programming," Operations Research, vol. 51, No. 6, pp. 850-865, 2003.

[9] M. Valenti, "Approximate Dynamic Programming with Applications in Multi-Agent Systems," Ph.D. dissertation, Massachusetts Institute of Technology, 2007.

[10] A. Smola, B. Scholkopf, "A Tutorial on Support Vector Regression," Statistics and Computing, vol. 14, pp. 199-222, 2004.

[11] B. Curry and P. Morgan, "Model selection in neural networks: Some difficulties," European Journal of Operational Research, vol. Volume 170, Issue 2, pp. p.567-577, 2006.

[12] R. Patrascu, "Linear approximations for factored markov decision processes," PhD Dissertation, University of Waterloo, Department of Computer Science, February 2004.

[13] A. S. B. Scholkopf, Learning with Kernels: Support Vector Machines, Regularization, Optimization, and Beyond. MIT Press, Cambridge, MA, 2002.

[14] C. Rasmussen and C. Williams, Gaussian Processes for Machine Learning. MIT Press, Cambridge, MA, 2006.

[15] J. Platt, "Using sparseness and analytic QP to speed training of support vector machines," in Advances in Neural Information Processing Systems, 1999, pp. 557-563.

[16] S. Keerthi, S. Shevade, C. Bhattacharyya, and K. Murthy, "Improvements to Platt's SMO algorithm for SVM classifier design," http://citeseer.ist.psu.edu/244558.html, 1999.

[17] C.-C. Chang and C.-J. Lin, LIBSVM: a library for support vector machines, 2001, Software available at http://www.csie.ntu.edu.tw/ cjlin/libsvm.

[18] R.S. Sutton, "Learning to Predict by the Methods of Temporal Differences," Machine Learning, vol. 3, pp. 9-44, 1988. 